The Astrophysical Journal, 689:883-888, 2008 December 20

(C) 2008. The American Astronomical Society. All rights reserved. Printed in U.S.A.

\title{
STAR FORMATION RATES IN LYMAN BREAK GALAXIES: RADIO STACKING OF LBGs IN THE COSMOS FIELD AND THE SUB- $\mu$ Jy RADIO SOURCE POPULATION ${ }^{1}$
}

\author{
C. L. Carillit, ${ }^{2}$ Nicholas Lee, ${ }^{2}$ P. Capak, ${ }^{3}$ E. Schinnerer,${ }^{4}$ K.-S. Lee, ${ }^{5}$ H. McCraken, ${ }^{6}$ M. S. Yun, ${ }^{5}$ \\ N. Scoville, ${ }^{3}$ V. Smolčić, ${ }^{3}$ M. Giavalisco, ${ }^{5}$ A. Datta, ${ }^{2}$ Y. Taniguchi, ${ }^{7}$ and C. Megan Urry ${ }^{8,9}$ \\ Received 2008 April 21; accepted 2008 August 1
}

\begin{abstract}
We present an analysis of the radio properties of large samples of Lyman break galaxies (LBGs) at $z \sim 3,4$, and 5 from the COSMOS field. The median stacking analysis yields a statistical detection of the $z \sim 3$ LBGs ( $U$-band dropouts), with a $1.4 \mathrm{GHz}$ flux density of $0.90 \pm 0.21 \mu \mathrm{Jy}$. The stacked emission is unresolved, with a size $<1$, or a physical size $<8 \mathrm{kpc}$. The total star formation rate implied by this radio luminosity is $31 \pm 7 \mathrm{M}_{\odot} \mathrm{yr}^{-1}$, based on the radio-FIR correlation in low-redshift star-forming galaxies. The star formation rate derived from a similar analysis of the UV luminosities is $17 M_{\odot} \mathrm{yr}^{-1}$, without any correction for $\mathrm{UV}$ dust attenuation. The simplest conclusion is that the dust attenuation factor is 1.8 at UV wavelengths. However, this factor is considerably smaller than the standard attenuation factor of $\sim 5$, normally assumed for LBGs. We discuss potential reasons for this discrepancy, including the possibility that the dust attenuation factor at $z \geq 3$ is smaller than at lower redshifts. Conversely, the radio luminosity for a given star formation rate may be systematically lower at very high redshift. Two possible causes for a suppressed radio luminosity are (1) increased inverse Compton cooling of the relativistic electron population due to scattering off the increasing CMB at high redshift or (2) cosmic-ray diffusion from systematically smaller galaxies. The radio detections of individual sources are consistent with a radio-loud AGN fraction of $0.3 \%$. One source is identified as a very dusty, extreme starburst galaxy (a "submillimeter galaxy").
\end{abstract}

Subject headings: galaxies: evolution — galaxies: formation — surveys

\section{INTRODUCTION}

The power of discovering high-redshift galaxies via the broadband dropout technique, i.e., Lyman break galaxies (LBGs), is now well established (Steidel et al. 1999, 2000). Using this technique, over a thousand galaxies have now been detected at $z>2$. Detailed studies show that these galaxies have stellar masses between $10^{10}$ and $10^{11} M_{\odot}$ and a (comoving) volume density at $z \sim 3$ of $\sim 0.005 \mathrm{Mpc}^{-3}$ (Giavalisco 2002).

A number of issues are still being investigated for LBGs. One important issue is deriving the total star formation rate. Besides model parameters such as the initial mass function (IMF) and star formation history, the dust correction in the UV remains under investigation. Steidel et al. (1999) originally estimated a typical UV dust attenuation factor of $\sim 5$, based on optical spectroscopy. Adelberger \& Steidel (2000) applied a similar method to a larger sample of LBGs, as well as observations at other wave bands (radio, submillimeter), and conclude that "the mean extinction at $1600 \AA$ for LBGs, a factor of 6 in our best estimate, could lie

\footnotetext{
${ }^{1}$ Based on observations in the COSMOS Legacy Survey including those taken on the HST, Keck, NRAO-VLA, Subaru, KPNO $4 \mathrm{~m}$, CTIO $4 \mathrm{~m}$, and CFHT $3.6 \mathrm{~m}$. The Very Large Array of the National Radio Astronomy Observatory is a facility of the National Science Foundation operated under cooperative agreement by Associated Universities, Inc.

2 National Radio Astronomy Observatory, P.O. Box 0, Socorro, NM 87801-0387.

${ }^{3}$ California Institute of Technology, MC 105-24, 1200 East California Boulevard, Pasadena, CA 91125.

${ }^{4}$ Max Planck Institut für Astronomie, Königstuhl 17, D-69117 Heidelberg, Germany.

${ }^{5}$ Department of Astronomy, University of Massachusetts, Amherst, MA.

${ }^{6}$ Institut d'Astrophysique de Paris, Paris, France.

7 Graduate School of Science and Engineering, Ehime University, Bunkyocho, Matsuyama 790-8577, Japan.

${ }^{8}$ Department of Astronomy, Yale University, New Haven, CT 06511.

9 Yale Center for Astronomy and Astrophysics, Yale University, P.O. Box 208121, New Haven, CT 06520.
}

between a factor of 5 and a factor of 9." More recently, Reddy \& Steidel (2004) have derived the average dust correction factors for UV-selected galaxies at $z \sim 2$ based on deep X-ray, radio, and optical spectroscopic studies of galaxies in the GOODS north field. They find that, for galaxies with total star formation rates $>20 M_{\odot} \mathrm{yr}^{-1}$, the dust attenuation factor is between 4.4 and 5.1 .

A second issue for LBGs is the AGN fraction. Shapley et al. (2003) found that $\sim 3 \%$ of LBGs at $z \sim 3$ have optical emission line spectra that are consistent with AGNs.

The Cosmic Evolution Survey (COSMOS), covering $2 \mathrm{deg}^{2}$, is a comprehensive study of the evolution of galaxies, AGNs, and dark matter as a function of their cosmic environment. The COSMOS field has state-of-the-art multiwavelength observations, ranging from the radio through the X-ray (Scoville et al. 2007; Capak et al. 2007). A major part of this study is to identify the largest samples of LBGs to date. In $\S 2$ we describe the LBG samples from the COSMOS survey based on $U-, B$-, and $V$-band dropout searches.

In this paper we consider the radio properties of these large samples of LBGs, similar to the study of $z=5.7$ Ly $\alpha$ emitting galaxies by Carilli et al. (2007). Observations of the COSMOS field have been done at $1.5^{\prime \prime}$ resolution (FWHM) at $1.4 \mathrm{GHz}$ with the Very Large Array (VLA; Schinnerer et al. 2007). We employ the new VLA image that has added integration time in the central $\sim 1^{\circ}$ (E. Schinnerer 2008, in preparation), giving an rms at the field center of $\sim 7 \mu \mathrm{Jy}_{\text {beam }}^{-1}$. In $\S 3.1$ we search for radio counterparts to individual LBGs, while in $\S 3.2$ we perform a stacking analysis to derive the statistical properties of the samples. In $\S 4$ we discuss the implications that these observations have on the questions of dust extinction and the AGN fraction in LBGs. We adopt a standard concordance cosmology.

\section{A CONSERVATIVE COSMOS LBG SAMPLE}

We have identified large samples of $U-, B-$, and $V$-band dropout galaxies using the COSMOS photometric data (Capak et al. 
TABLE 1

Cosmos Dropout Selection

\begin{tabular}{cccc}
\hline \hline Source & Color & Color & Color \\
\hline$U$-band dropouts .............. & $\left(u^{\prime}-B\right) \geq 0.7\left(B-i^{\prime}\right)+0.7$ & $\left(u^{\prime}-B\right) \geq 1.4$ & $\left(B-i^{\prime}\right) \leq 2.5$ \\
$B$-band dropouts............... & $(B-V) \geq 0.7\left(V-i^{\prime}\right)+0.7$ & $(B-V) \geq 1.0$ & $\left(V-i^{\prime}\right) \leq 2.5$ \\
$V$-band dropouts ............... & $\left(V-r^{\prime}\right) \geq 0.3\left(r^{\prime}-i^{\prime}\right)+1.0$ & $\left(V-r^{\prime}\right) \geq 1.4$ & $\left(r^{\prime}-i^{\prime}\right) \leq 3.0$ \\
\hline
\end{tabular}

2007). For an initial, robust analysis of the radio properties of high- $z$ LBGs, we have adopted a series of conservative criteria to identify LBGs at the different redshifts. The $U-, B$-, and $V$-band drop selection criteria are given in Table 1 , where $u^{\prime}=$ CFHT $u$, $i^{\prime}=$ Subaru $i$, and $r^{\prime}=$ Subaru $r$ (P. Lee et al. 2008, in preparation).

It is well documented that LBG samples can include contaminants (see Steidel et al. 2000; Capak et al. 2004; Bouwens et al. 2007). In the $U$ - and $B$-band dropout selection, the contamination comes from $A$ and $M$ stars along with $z<0.3$ star-forming galaxies, while the $V$-band dropout selection is contaminated with $\mathrm{M}$ and T stars along with $1.5<z<2.5$ obscured galaxies. Since these contaminating objects can be sources of radio emission, we take several steps to produce a clean sample of high-redshift objects. We begin by removing all ACS point sources with a staror AGN-like spectral energy distribution using the star/galaxy/ AGN classifier described in Robin et al. (2007). In addition, we remove all stars identified by a $B z K$ diagram (Daddi et al. 2007) at $K_{\mathrm{AB}}<22$; this removes very red stars that may not be identified by the Robin et al. (2007) classifier. We then use the $B P Z$ photometric redshifts from Mobasher \& Mazzei (2000) to remove objects with a best-fit phot- $z$ at $z<2.5, z<3.5$, and $z<4.5$ for the $U-, B$-, and $V$-band dropouts, respectively. Finally, we remove objects with a $>5 \%$ probability of being at $z \leq 1$.

The resulting sample is largely clear of contaminants. Redshifts were obtained with DEIMOS on Keck II using the 830 line $\mathrm{mm}^{-1}$ grating and 3.5-4 hr of integration time for a sample of colorselected objects with $i<26$ and $0.6<z<1.4$ or $z>4$ (P. Capak et al. 2008, in preparation). The spectroscopic sample matches two $U$-band dropouts, $18 \mathrm{~B}$-band dropouts, and seven $V$-band dropouts in our cleaned LBG sample. Of these objects, only one $V$-band dropout object, which is a faint M star, is not in the expected redshift range, yielding a contamination fraction of $4 \% \pm$ $4 \%$. However, the $U$-band dropout sample was not specifically targeted, so the constraint on the contamination fraction for these objects is weak. Of the remaining 26 objects at $z>3$, one $B$-band dropout shows an AGN signature, implying a residual AGN fraction of $4 \% \pm 4 \%$.

Although our sample is clean, it is not complete. In general, the phot- $z$ cut creates a bias toward galaxies that have good photometry and that closely match the expected UV-optical spectral templates used for the photometric redshifts. Objects with unusual spectra, such as AGN/galaxy composites, or objects with reddening that does not conform to the Calzetti (1997) or Milky Way laws, will likely be excluded. Furthermore, we have ex- plicitly excluded optically bright broad-line AGNs, and the probability requirements systematically bias us against faint $(i<25)$ objects. The flux bias occurs because faint objects have broad probability distributions due to their poor photometric measurements.

We are also potentially biased against heavily obscured objects that will have fainter UV fluxes and hence poor phot-z fits. An effort to use intermediate band photometry (Ilbert et al. 2008, in preparation) and a systematic deep spectroscopic follow-up (Lilly et al. 2007; P. Capak et al. 2008, in preparation) is under way to fully characterize this sample and understand the biases.

Table 2 summarizes the current samples. Column (2) indicates the redshift range implied for a given dropout band. Column (3) shows the number of sources in the sample. Even with these conservative selection criteria, we identify thousands of highredshift LBGs due to the very wide COSMOS field. In this initial study, we feel it is important to work with such a conservative sample of sources, in order to make robust conclusions concerning the radio properties of LBGs.

For reference, the radio luminosity density at a rest-frame frequency of $1.4 \mathrm{GHz}$, for a source with $S_{1.4 \mathrm{GHz}}=60 \mu \mathrm{Jy}$, assuming a spectral index of -0.8 (Condon 1992), at $z \sim 3$ is $L_{1.4 \mathrm{GHz}}=$ $3 \times 10^{31} \mathrm{ergs} \mathrm{s}^{-1} \mathrm{~Hz}^{-1}$. For comparison, the nearby radio AGN source M87 has $L_{1.4 \mathrm{GHz}}=9 \times 10^{31} \mathrm{erg} \mathrm{s}^{-1} \mathrm{~Hz}^{-1}$, while the luminous starburst galaxy Arp 220 has $L_{1.4 \mathrm{GHz}}=4 \times 10^{30} \mathrm{erg} \mathrm{s}^{-1}$ $\mathrm{Hz}^{-1}$. In M87, the radio emission is powered by a relativistic jet emanating from the supermassive black hole with $M_{\mathrm{BH}} \sim$ $2.5 \times 10^{9} M_{\odot}$ (Gebhardt et al. 2000), while in Arp 220 the radio emission is driven by star formation with a total star formation rate $\sim 500 M_{\odot} \mathrm{yr}^{-1}$ (Carilli \& Yun 2000; Yun \& Carilli 2001). Even at the sensitivity of the COSMOS survey, we are limited to detecting either radio AGN sources or extreme starburst galaxies, i.e., galaxies with star formation rates greater than a few thousand $M_{\odot} \mathrm{yr}^{-1}$.

\section{THE RADIO PROPERTIES OF LYMAN BREAK GALAXIES}

\subsection{Individual Sources}

We have searched for radio emission from each of the LBGs in the COSMOS field. At each position we determine the surface brightness and the rms noise in the region. The median rms in the LBG fields was $\sim 15 \mu \mathrm{Jy}_{\text {beam }}{ }^{-1}$ for all three samples, implying a median $4 \sigma$ detection limit of $60 \mu \mathrm{Jy}_{\text {beam }}^{-1}$. Our radio images

TABLE 2

Statistics of Lyman Break Sample and Results of the $4 \sigma$ Counterpart Search

\begin{tabular}{|c|c|c|c|c|c|}
\hline $\begin{array}{c}\text { Band } \\
(1)\end{array}$ & $\begin{array}{l}\text { Redshift } \\
\text { (2) }\end{array}$ & $\begin{array}{l}\text { Number of LBGs } \\
\text { (3) }\end{array}$ & $\begin{array}{c}\text { Number of } 4 \sigma \text { Sources } \\
\text { (4) }\end{array}$ & $\begin{array}{l}\text { Corrected Number of Sources } \\
\text { (5) }\end{array}$ & $\begin{array}{c}\text { Median Flux Density } \\
(\mu \mathrm{Jy}) \\
(6)\end{array}$ \\
\hline$U$ & $2.5-3.5$ & 6457 & $43(0.67 \%)$ & $24(0.38 \%)$ & $0.90 \pm 0.21$ \\
\hline В & $3.5-4.5$ & 1447 & $8(0.55 \%)$ & $5(0.34 \%)$ & $0.83 \pm 0.42$ \\
\hline$V$ & $>4.5$ & 614 & $5(0.81 \%)$ & $2(0.33 \%)$ & $1.72 \pm 0.68$ \\
\hline
\end{tabular}


have a synthesized beam FWHM $=1.5^{\prime \prime}$; hence, we have searched for radio sources brighter than $4 \sigma$, within $1^{\prime \prime}$ radius of the LBG optical position. Column (4) in Table 2 lists the number of $4 \sigma$ radio counterparts in each sample. Roughly, for each dropout band, we detect radio counterparts for $\sim 0.6 \%$ of the UV sources.

We need to correct for the number of detections we expect by chance in such a survey, either due to image noise, or faint sources unrelated to the LBG. We calculate this correction factor by performing searches with identical criteria as for the LBG positions, but at random positions selected to be $20^{\prime \prime}$ away from the coordinates of the LBG samples. This should give us a random sampling of the COSMOS field, with noise statistics that match the LBG regions. We find $194 \sigma$ random detections in the $U$-band dropout fields, four for the $B$-band dropouts, and three for the $V$-band dropouts. These correspond to $0.29 \%, 0.28 \%$, and $0.49 \%$, respectively. We apply these corrections in column (5) of Table 2.

Overall, we detect radio counterparts to $\sim 0.6 \%$ of the UV sources, with the expected number of detections at random being $\sim 0.3 \%$. These results imply that only a small fraction, $\sim 0.3 \%$, of the LBGs, are radio sources brighter than $\sim 4 \sigma$, where, on average, $4 \sigma \sim 60 \mu \mathrm{Jy}$.

\subsection{A Stacking Analysis}

We investigate the statistical radio properties of the LBGs through a stacking analysis. We adopt the median stacking method used by White et al. (2007) in their stacking analysis based on the FIRST radio survey. White et al. perform detailed calculations that show that a median stacking analysis is superior to a mean stacking, since it is robust to small numbers of bright sources, and it does not require any maximum allowed flux density cutoff prior to stacking. Moreover, they show that "in a limit where almost all of the values in the sample are small compared to the noise, it is straightforward to interpret the median stack measurements as representative of the mean for the population." The COSMOS sample obeys this criterion.

Figure 1 shows the images derived by a median stacking of all the sources in each dropout band. These images are derived by extracting subimages centered around each LBG with a size of $18^{\prime \prime} \times 18^{\prime \prime}$. We then "stack" these subimages, aligned at the center (on-source) position, and derive the median surface brightness at each pixel position. The off-source pixels serve as test positions for a random stacking analysis. We find that the mean values for the off positions equal zero, to within the noise, as expected for random positions. We also derive the statistical noise of the analysis from these off-positions.

Column (6) in Table 2 lists the peak surface brightnesses derived at the stacked LBG position, plus the rms off-source noise, for each sample. We obtain a clear detection of radio emission in the $U$-band dropout sample, with a value of $0.9 \pm 0.21 \mu \mathrm{Jy}$. The other bands show marginal detections, albeit at $<3 \sigma$ value, and hence we consider these upper limits.

Our results are consistent with the upper limit found by Ivison et al. (2007), who performed a $1.4 \mathrm{GHz}$ stacking analysis of $107 z \sim 3$ LBGs in the extended Groth strip (the AEGIS survey), for which they obtained a median value of $-0.6 \pm 2.3 \mu \mathrm{Jy}$.

The stacked source is unresolved, with a size $<1$. The implied physical size is $<8 \mathrm{kpc}$; hence, the typical source must be galaxy size, or smaller.

\section{DISCUSSION}

\subsection{The AGN Fraction and Massive Starbursts}

At the sensitivity limits of current deep radio surveys, such as COSMOS, detection of individual sources at $z>2$ is limited to either extreme starbursts (star formation rates $>1000 M_{\odot} \mathrm{yr}^{-1}$ ), or radio jet sources with luminosities comparable to M87.

After correcting for random detections, we obtain a detection rate of $\sim 0.3 \%$. Shapley et al. (2003) find an AGN fraction in LBG samples of $\sim 3 \%$, based on optical spectroscopy. If all of our detected sources are radio AGNs, then the implied radio-loud fraction is $\sim 10 \%$. Interestingly, a value of $10 \%$ is the canonical value for radio-loud AGNs based on studies of nearby galaxies (e.g., Ivezic et al. 2002; Petric et al. 2007).

We emphasize caution in interpreting our results on the radioloud fraction of AGNs at high redshift, for a number reasons. First, in a recent comparison of the FIRST and SDSS surveys, Jiang et al. (2007) find that the radio-loud fraction likely depends on both optical luminosity and redshift. This study is not directly comparable to our COSMOS study, since they were limited to much more luminous sources at high redshift, by about 2 orders of magnitude in the radio.

Second, the above analysis does not consider the possibility that some of the $0.3 \%$ of the radio detections are extreme, highly obscured starbursts, comparable to the submillimeter galaxies. Indeed, in a companion paper, we present the discovery of a submillimeter galaxy in our radio-selected LBG COSMOS sample (Capak et al. 2008). The radio-detected LBG has a spectroscopic redshift of $z=4.5$ and a radio flux density of $45 \pm 10 \mu \mathrm{Jy}$. Thermal emission from warm dust is detected from this galaxy at $250 \mathrm{GHz}$ using the MAMBO bolometer camera, with a flux density of $3.4 \pm 0.7 \mathrm{mJy}$. This source is the first submillimeter galaxy yet identified (spectroscopically) at $z>4$ (Capak et al. 2008).

And third are the caveats mentioned in $\S 2$ concerning our conservative source selection criteria, for example, excluding obvious optically bright broad-line AGNs.

\subsection{The Sub- $\mu J y$ Radio Source Population}

The unprecedented size of the COSMOS LBG sample has allowed us to reach sub- $\mu \mathrm{Jy}$ sensitivity levels in the stacking analysis of the LBG samples. We obtain a clear statistical detection of $S_{1.4 \mathrm{GHz}}=0.90 \pm 0.21 \mu \mathrm{Jy}$ for the $U$-band $(z \sim 3)$ dropouts. The implied rest-frame $1.4 \mathrm{GHz}$ luminosity density is $L_{1.4 \mathrm{GHz}}=5.1 \times 10^{29} \mathrm{erg} \mathrm{s}^{-1} \mathrm{~Hz}^{-1}$, assuming a spectral index of -0.8 (Condon 1992).

Assuming that the radio emission is driven by star formation, we can derive a total star formation rate $\left(0.1-100 M_{\odot}\right)$ from the rest-frame $1.4 \mathrm{GHz}$ luminosity density. There are a number of recent calibrations of this relationship for nearby galaxies using, for example, the IRAS and NVSS surveys. These studies adopt the star formation rates derived from the far-IR emission based on the relations in Kennicutt (1998), assuming a Salpeter IMF, and then calibrate the radio conversion factor using the tight radio to far-IR correlation for star-forming galaxies. We adopt the conversion factor from the study of Yun et al. (2001):

$$
\mathrm{SFR}=(5.9 \pm 1.8) \times 10^{-29} L_{1.4 \mathrm{GHz}} M_{\odot} \mathrm{yr}^{-1}
$$

where $L_{1.4 \mathrm{GHz}}$ is in $\mathrm{erg} \mathrm{s}^{-1} \mathrm{~Hz}^{-1}$. From this, we calculate a mean star formation rate of $31 \pm 7 M_{\odot} \mathrm{yr}^{-1}$. Note that the conversion factor above is within $10 \%$ of that derived by Bell (2003).

The mean observed UV luminosity at a rest-frame wavelength of $1600 \AA$ of the $U$-band dropout sample is $L_{2000} \AA=$ $1.2 \times 10^{29} \mathrm{erg} \mathrm{s}^{-1} \mathrm{~Hz}^{-1}$ (P. Capak et al. 2008, in preparation). Using equation (1) of Kennicutt (1998) for the relationship between UV luminosity and total star formation rate, we derive a 

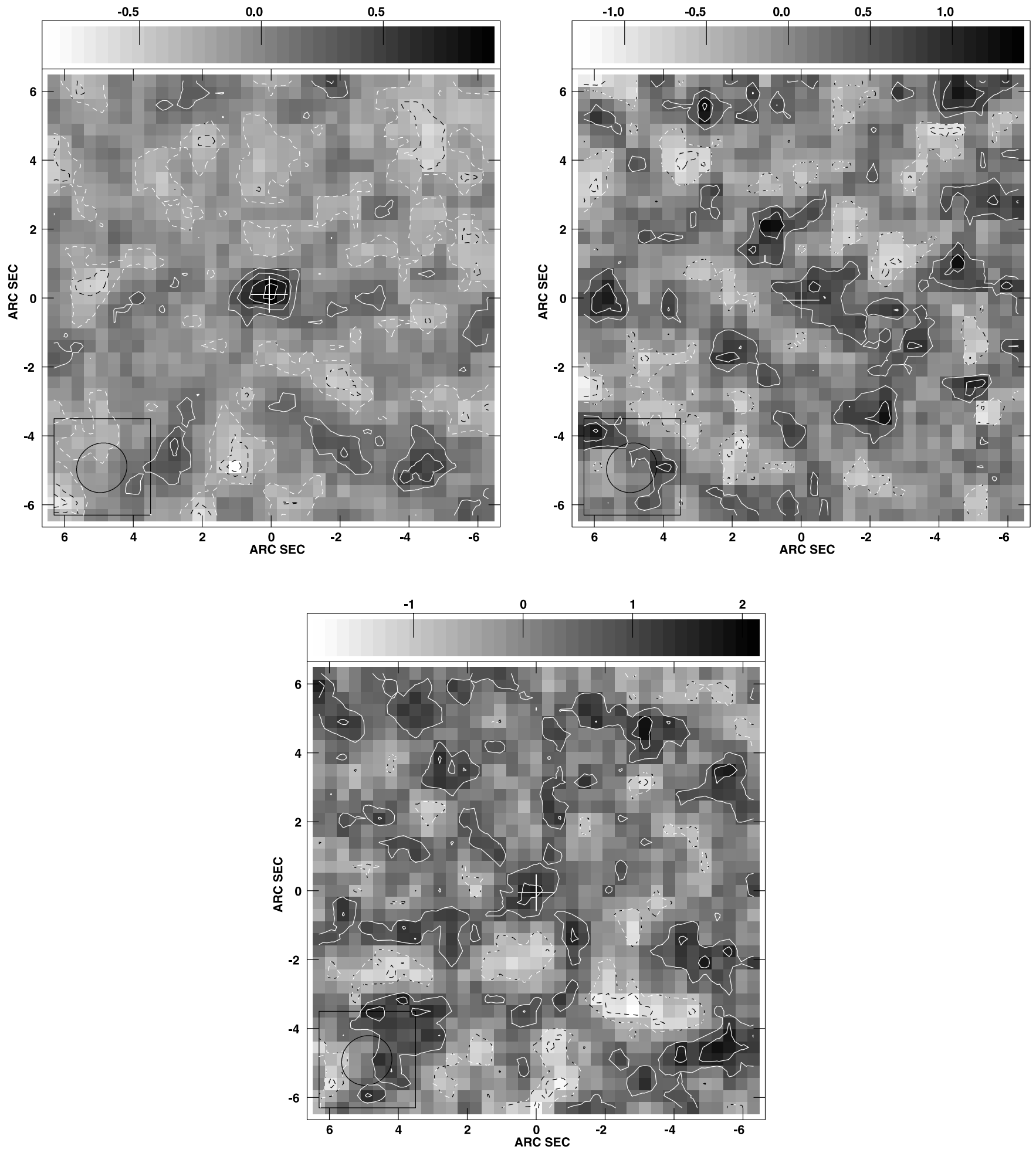

FIG. 1.-Images at $1.4 \mathrm{GHz}$ of the median stacking results for the different dropout samples. In each case, the contour levels are $-3,-2,-1,1,1,2,3$, and 4 times the rms value in the image, and the plus sign indicates the source stacking position. The synthesized beam FWHM is shown in the lower left corner. Top left: $U$-band dropout result, with $\mathrm{rms}=0.21 \mu \mathrm{Jy}$ beam $^{-1}$. Top right: $B$-band dropouts result, with $\mathrm{rms}=0.42 \mu \mathrm{Jy}_{\text {beam }}{ }^{-1}$. Bottom: $V$-band dropouts result, with rms $=0.68 \mu \mathrm{Jy}$ beam ${ }^{-1}$.

total star formation rate of $17 M_{\odot} \mathrm{yr}^{-1}$, uncorrected for dust attenuation.

The ratio of radio-derived star formation rate to UV-derived star formation rate is $1.8 \pm 0.4$. The simplest conclusion is that the UV emission is attenuated by dust by a factor of 1.8 . However, this factor is considerably smaller than the standard factor of $\sim 5$ adopted for LBGs (see $\S 1$ ). We consider some possible reasons for this difference.
One possibility is that the dust attenuation factor for the $z \sim 3$ COSMOS LBG sample is indeed smaller than those for other LBG samples. Most of the studies of dust attenuation of LBG galaxies have been at $z<3$ (see $\S 1$ ), and hence it is possible that at higher redshift the dust attenuation factor decreases. An argument against this decrease is the recent X-ray and optical study of a sample of LBGs at $z \sim 3$ by Nandra et al. (2002), who also derive a dust attenuation factor of $\sim 5$. We also reemphasize that 
the COSMOS LBG sample analyzed herein deselected sources with poor phot- $z$ fits, which can occur for very heavily obscured objects $(\S 2)$.

On the other hand, Wilkins et al. (2008) present a detailed comparison of the buildup of stellar mass in galaxies versus the cosmic star formation rate density. They conclude that there is a discrepancy between the rate of stellar mass creation and the star formation rates derived from the UV luminosities at high redshift, if one assumes the standard factor of $\sim 5$ dust attenuation. The discrepancy is in the sense that the UV-derived star formation rates are too high. They find that the peak in the discrepancy occurs at $z \sim 3$, and they suggest that the UV-derived star formation rates at this redshift may be overestimated by a factor of $\sim 4$. They also point out that "this large deviation at high redshift offers an explanation for why the integrated star formation history implies a local stellar mass density in excess of that measured."

A second possibility is that the conversion factor of radio luminosity to star formation rate is higher in the $z \sim 3 \mathrm{LBG}$ COSMOS sample than has been derived for low-redshift galaxies. Radio studies of $24 \mu \mathrm{m}$ selected galaxies by Appleton et al. (2004) imply that the radio conversion factor is constant out to $z \sim 1$, i.e., that the radio-FIR correlation is constant out to this redshift. Reddy \& Steidel (2004) extend this conclusion out to $z \sim 2$ in their extensive study of UV-selected galaxies. Most recently, Ibar et al. (2008) conclude that the radio conversion factor remains constant out to $z \sim 3$, using a $24 \mu \mathrm{m}$ source sample with redshifts from the SXDF. However, given the need for individual source detections in the radio, their $z \sim 3$ radio sources have star formation rates about 2 orders of magnitude larger than the stacking results presented herein, and hence a direct comparison is problematic.

One physical reason why we might expect the radio conversion factor to diverge at the highest redshifts is increased relativistic electron cooling due to inverse Compton scattering off the cosmic microwave background (CMB). The ratio of relativistic electron energy losses due to synchrotron radiation, to energy losses due to inverse Compton radiation, equals the ratio of the energy density in the magnetic field to that in the photon field. The energy density in the CMB increases as $U_{\mathrm{CMB}}=4.2 \times$ $10^{-13}(1+z)^{4} \mathrm{ergs} \mathrm{cm}^{-3}$. Figure 2 shows a comparison of $U_{\mathrm{CMB}}$ with the typical energy densities in the magnetic fields in different regions in galaxies. We show the range of fields considered typical for spiral arms $(\sim \mathrm{a}$ few $\mu \mathrm{G})$, and for starburst galaxy nuclei (of order $100 \mu \mathrm{G}$; Beck et al. 1994; see review by Beck 2005). The important point is that inverse Compton losses off the CMB will dominate synchrotron losses in a typical ISM at $z \geq 0.5$ and dominate in starburst nuclei at $z \geq 4$.

We note that inverse Compton losses will not affect the thermal electrons responsible for free-free emission from starforming galaxies. Such free-free emission may dominate the total radio emission from galaxies at rest frequencies between roughly 40 and $100 \mathrm{GHz}$ (Condon 1992) and hence become an important factor in radio continuum studies of very high redshift galaxies.

A depressed radio luminosity for a given star formation rate could also arise if the $z \sim 3$ LBGs are systematically smaller galaxies. The standard model that produces the radio-FIR correlation (Condon 1992) requires a cosmic-ray processing box size $\geq 1 \mathrm{kpc}$. It has been observed that dwarf galaxies at low redshift depart from the radio-FIR correlation by about a factor of 2 , in the sense of being radio-underluminous. The hypothesis is that the cosmic rays diffuse out of the galaxy on timescales shorter

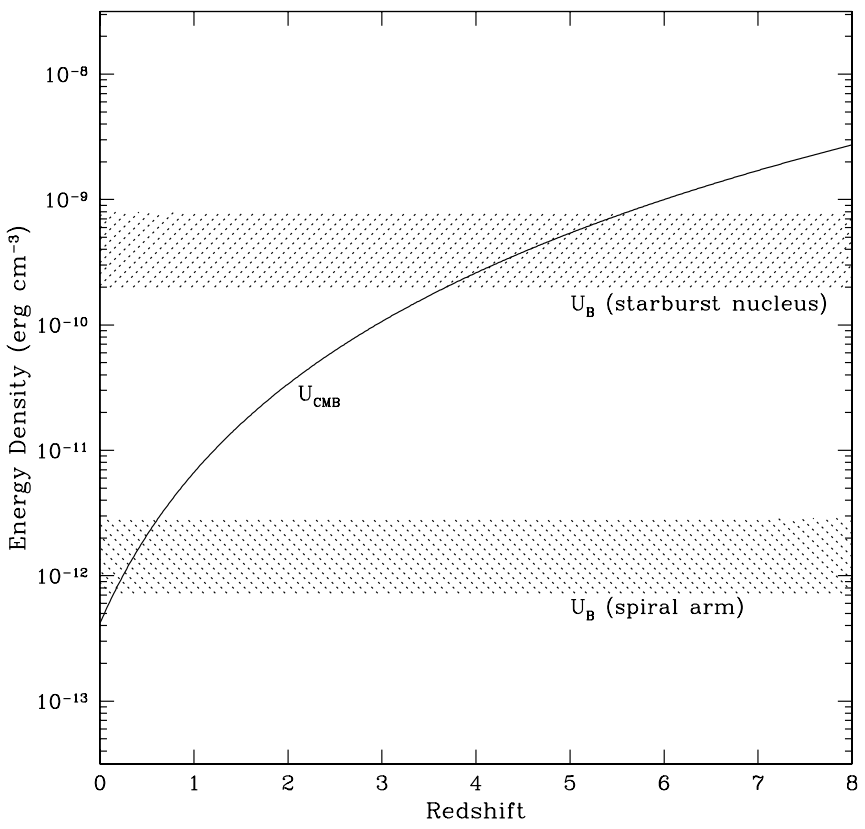

FIG. 2.-Energy density in the CMB as a function of redshift, plus shaded regions showing typical ranges for magnetic fields in spiral arms, and starburst galaxy nuclei.

than those required to maintain the standard radio-FIR correlation (Yun et al. 2001). Giavalisco (2002) describes high- $z$ LBGs as having typical half-light radii of 4-7 kpc, significantly larger than typical dwarf galaxies. However, he points out that "frequently the galaxies have disturbed or fragmented morphologies, with one bright core, or multiple knots embedded in diffuse nebulosity, reminiscent of merger events."

In this paper, we report the first robust statistical (median) detection of sub- $\mu \mathrm{Jy}$ radio emission from $\mathrm{LBG}$ galaxies at $z \sim 3$. This detection was made possible by the very wide area, and depth, of the COSMOS field. While our physical interpretation of the result remains inconclusive, there are a number of future studies we are pursuing to address the interesting questions raised concerning the UV attenuation factor for LBGs, and the radio luminosity to star formation rate conversion factor, at $z \geq 3$. The most important study involves obtaining optical spectra of a large sample of LBGs from the COSMOS sample. Spectra will elucidate the nature of the sources and allow for a study of the dust correction factor as a function of galaxy type (AGN, starburst, elliptical, etc.). Also, the selection criteria for this LBG sample were very conservative $(\S 2)$. We will further refine (and increase) our LBG samples using the COSMOS multiband photometry and perform statistical analyses of the radio properties as a function of, for example, stellar mass, or $A_{V}$. We are also exploring the IR properties of these samples with Spitzer. Such IR studies have particular relevance in light of the results of Ivison et al. (2007), who find that for the seven IR luminous $z \sim 3$ LBGs in their AEGIS sample $\left(S_{24 \mu \mathrm{m}}>60 \mu \mathrm{Jy}\right)$, the median $1.4 \mathrm{GHz}$ flux density is $44 \mu \mathrm{Jy}$, comparable to that expected for submillimeter galaxies. Lastly, unambiguous identification of radio AGNs using, for example, optical spectra, will provide input into faint radio source population models that are being generated in the context of planning for future, large area radio telescopes (Wilman et al. 2008). The results presented herein indicate that future deep (sub- $\mu \mathrm{Jy}$ ), wide field surveys with the Expanded Very Large Array, will routinely detect the radio emission from individual LBGs out to $z \sim 3$. 
The HST COSMOS Treasury program was supported through NASA grant HST-GO-09822. We gratefully acknowledge the contributions of the entire COSMOS collaboration consisting of more than 70 scientists. More information on the COSMOS survey is available at http://www.astro.caltech.edu/cosmos. C. C. thanks the Max-Planck-Gesellschaft and the Humboldt-Stiftung for support through the Max-Planck-Forschungspreis. We thank the referee for helpful comments.
Adelberger, K. L., \& Steidel, C. C. 2000, ApJ, 544, 218

Appleton, P. N., Fadda, D. T.; Marleau, F., et al. 2004, ApJS, 154, 147

Beck, R. 2005, in AIP Conf. Proc. 784, Magnetic Fields in the Universe: From Laboratory and Stars to Primordial Structures, ed. E. M. de Gouveia Dal Pino, G. Lugones, \& A. Lazarian (New York: AIP), 343

Beck, R. Carilli, C. L., Holdaway, M. A., \& Klein, U. 1994, A\&A, 292, 409 Bell, E. 2003, ApJ, 586, 794

Bouwens, R. J., Illingworth, G. D., Franx, M., \& Ford, H. 2007, ApJ, 670, 928

Capak, P., et al. 2004, AJ, 127, 180

$$
\text { 2007, ApJS, 172, } 99
$$$$
\text { 2008, ApJ, 681, L53 }
$$

Carilli, C. L., \& Yun, M. S. 2000, ApJ, 530, 618

Carilli, C. L., et al. 2007, ApJS, 172, 518

Calzetti, D. 1997, AJ, 113, 162

Condon, J. 1992, ARA\&A, 30, 575

Daddi, E., et al. 2007, ApJ, 670, 156

Gebhardt, K., et al. 2000, ApJ, 539, L13

Giavalisco, M. 2002, ARA\&A, 40, 579

Ibar, E., et al. 2008, MNRAS, 386, 953

Ivezic, Z., et al. 2002, AJ, 124, 2364

Ivison, R. J., et al. 2007, ApJ, 660, L77

Jiang, L., et al. 2007, ApJ, 656, 680

\section{REFERENCES}

Kennicutt, R. C., Jr. 1998, ARA\&A, 36, 189

Mobasher, B., \& Mazzei, P. 2000, A\&A, 363, 517

Nandra, K., et al. 2002, ApJ, 576, 625

Petric, A., Carilli, C. L., Bertoldi, F., Beelen, A., Cox, P., \& Omont, A. 2006, AJ, 132, 1307

Reddy, N., \& Steidel, C. 2004, ApJ, 603, L13

Robin, A. C., et al. 2007, ApJS, 172, 545

Schinnerer, E., et al. 2007, ApJS, 172, 46

Scoville, N., et al. 2007, ApJS, 172, 38

Shapley, A., Steidel, C., Pettini, M., \& Adelberger, K. 2003, ApJ, 588, 65

Steidel, C. C., Adelberger, C., Giavalisco, M., Dickinson, M., \& Pettini, M. 1999, ApJ, 519, 1

Steidel, C., Adelberger, K., Shapley, A., Pettini, M., Dickinson, M., \& Giavalisco, M. 2000, ApJ, 532, 170

White, R. L., Helfand, D. J., Becker, R. H., Glikman, E., \& de Vries, W. 2007, ApJ, 654, 99

Wilkins, S., Trentham, N., \& Hopkins, A. 2008, MNRAS, 385, 687

Wilman, R. J., Miller, L., Jarvis, M., Mauch, T., Levrier, F., et al. 2008, MNRAS, 388, 1335

Yun, M. S., \& Carilli, C. L. 2002, ApJ, 568, 88

Yun, M. S., Reddy, N., \& Condon, J. 2001, ApJ, 554, 803 